\title{
Multi-robot Voronoi tessellation based area partitioning algorithm study
}

https://doi.org/10.1515/pjbr-2018-0014

Received December 10, 2017; accepted June 19, 2018

\begin{abstract}
This article is focused on the multi-robot applied Voronoi tessellation based area partitioning algorithm operation and properties. The selection of main parameters of the algorithm is covered and explanation of the algorithm functionality is given. The subspace area equality and the operation time are taken as main measures of the algorithm operation for experiments where a dependency on the border shape and structure and a number of robots in the group are considered. The experimental data, gained during the study, is summarized mainly in a graphical way.
\end{abstract}

Keywords: mobile autonomous robots, cooperative behavior, collective behavior, collaborative multi-robot task, Voronoi tessellation, area partitioning, simulation

\section{Introduction}

In the paper, we present a novel architecture for a fully distributed tessellation algorithm and study the most important algorithm properties and its parametrization.

Multi-robot topics and collaborative robotics are gaining the interest of both theoretical and applied science in recent years. The application for the multi-robot activity coordination considered in the paper can be widely applied since it resolves a general problem of coordination in the area of operation. Nevertheless, besides the particular application in the paper, we would like to mention a few applicable examples:

- area coverage - cleaning tasks [1], resource foraging;

- autonomous vehicle control [2];

\footnotetext{
*Corresponding Author: Vladimir Alexandrov: National Research University "Moscow Power Engineering Institute", Russia; RobArt GmbH, E-mail: vladimir@alexandrov.email

Konstantin Kirik: National Research University "Moscow Power Engineering Institute", Russia; T-Platforms

Alexander Kobrin: National Research University "Moscow Power Engineering Institute", Russia
}

- patrolling on a given area [3];

- distributed sensing and networking using autonomous mobile objects [4].

Resources of the robotic group $[5,6]$ during the operation in a closed environment are used not only for the tasksolving algorithm implementation, but as well on:

- conflict, collision and resolution coordination;

- cooperative behavior for the multi-robot solution optimization.

It is important to mention, that the size of a robot relatively to the environment reference size matters. The bigger a robot relatively to an environment, the higher payload for the computational subsystem by the coordination and conflict resolution tasks is generated. There is a number of ways how one can resolve conflicts between group members during the task progression. Multi-agent trading cooperation [7], centralized guidance [8], collective swarm coordination approach [9] are among the most modern and advanced techniques for a robotic group conflict management. However, most of them either applicable for centralized systems only (centralized guidance) or cannot exclude collision during the operation, but resolves collisions once they happened.

This article describes another method - a way of coordination based on area partitioning, which structures the interaction and thus minimize number of conflicts between members of the group. During the research of multirobot area coverage algorithms authors found an interesting idea of building a tessellation over the area of operation prior to the actual coverage routine. The main advantage of the proposed method is a continuous separation of group members by virtual borders that are generated from the very beginning and being updated through the course of the action. This method would deliver an appropriate performance only when areas are similar or same size. In other words, it is important to make sure, that after partitioning each robot has same portion of work as others. Voronoi tessellation has been used in many similar applications [10-14], but there were several challenges connected to a particular application of the given method: 
1. System architecture in case of fully distributed algorithm run;

2. Creating a tessellation for the multi-robot system and parsing vertices targeted infinity;

3. Parametrization of the algorithm;

4. Extensive testing/simulation for different initial conditions in order to understand properties of the algorithm. Since the best case of having equal areas for each robot may not be always possible, answering the question of how similar areas of tessellation regions are becomes very important.

There are several papers dedicated to multi-robot Voronoi tessellation-based algorithms for different applications. Multi-robot Voronoi tessellation is considered in [10] as a control algorithm for agent-based sensor network. Similar algorithm is described in [11] where it is applied to an area coverage problem in a non-convex environment with obstacles. However, it remains unclear how the outside borders are handled during the run in both cases as well as the stability of the operation depending on border shape and overall system organization.

The main motivating aspects for the current work are:

1. The need of a fully distributed architecture capable of running the Voronoi-based tessellation routine, despite of having an existing drawback of required positioning information by each robot;

2. The parametrization/optimization required for the algorithm to succeed;

3. gaining missed clarity on how good in terms of a work split the tessellation is, i.e. how far are the resulting areas apart.

Building a solid algorithm applicable to a broad spectrum of convex-shaped environments utilizes the infinitylocated Voronoi vertices parsing and the universal polygon clipping routine and thus generalizes the algorithm to any polygon-defined operational area. While the algorithm works for the wide spectrum of input data, it cannot be called general, since it is developed under assumptions that robot positioning information available for each robot and the definition of a convex operation area is given by a polygon with finite number of vertices.

The paper is organized as follows: in section 2 the algorithm and the architecture of the control system are described. The way how the update period and the positioning precision is fixed for the algorithm is explained in section 3. This is followed by the description of the experiments for different initial conditions in section 4, where the most important properties of algorithm are considered. In section 5 a summary of the main outcome of the work and implementation drawbacks along with future work ideas are given.

\section{The algorithm}

The algorithm is executed for the robotic group $\boldsymbol{G}$ operating on the territory $\boldsymbol{Q}$ (shown in Figure 1). The algorithm is based on the Voronoi tessellation routine [15] and utilizes an interconnection via so-called "blackboard" shared memory distributed over the local group memories.

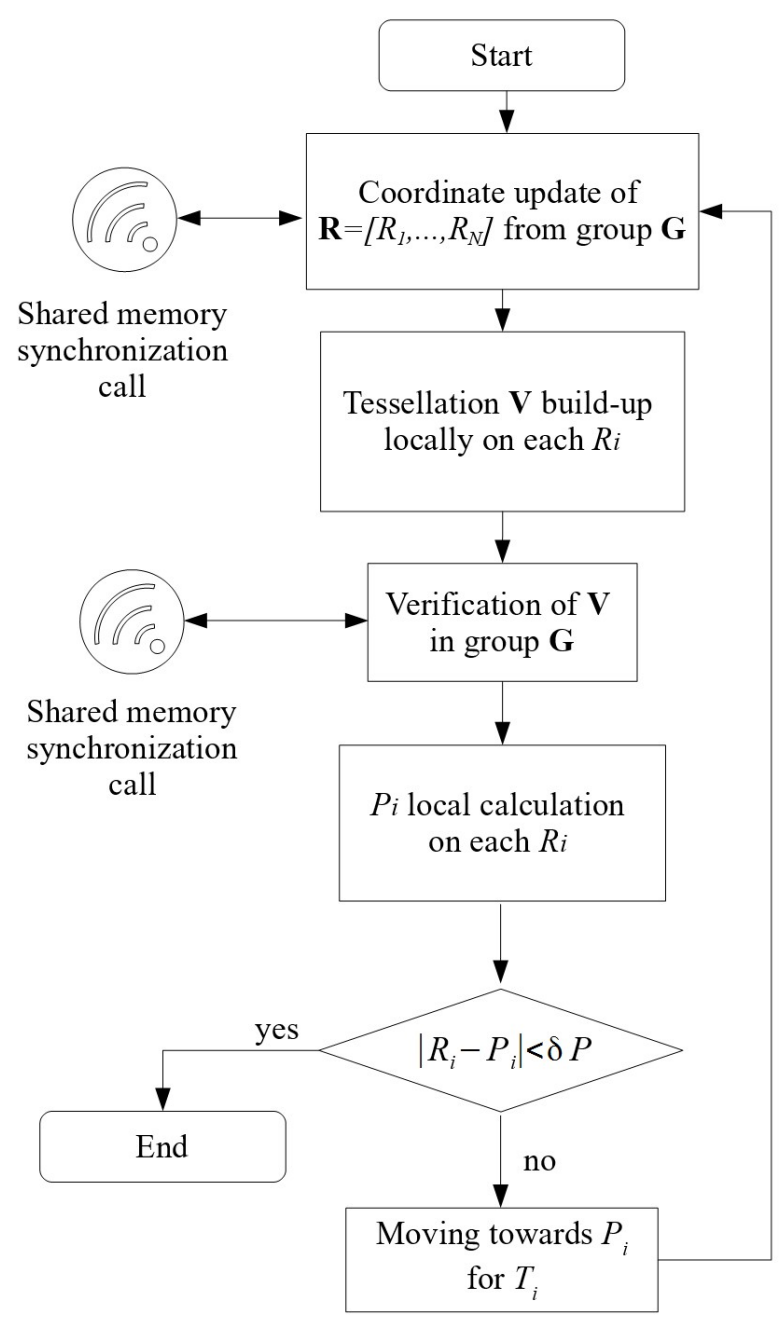

Figure 1: Voronoi multi-robot tessellation algorithm.

System architecture supporting the algorithm is shown in Figure 2. Local memories of robotic units are continuously synchronizing with each other and thus the memory area can be treated as a virtual shared storage. 


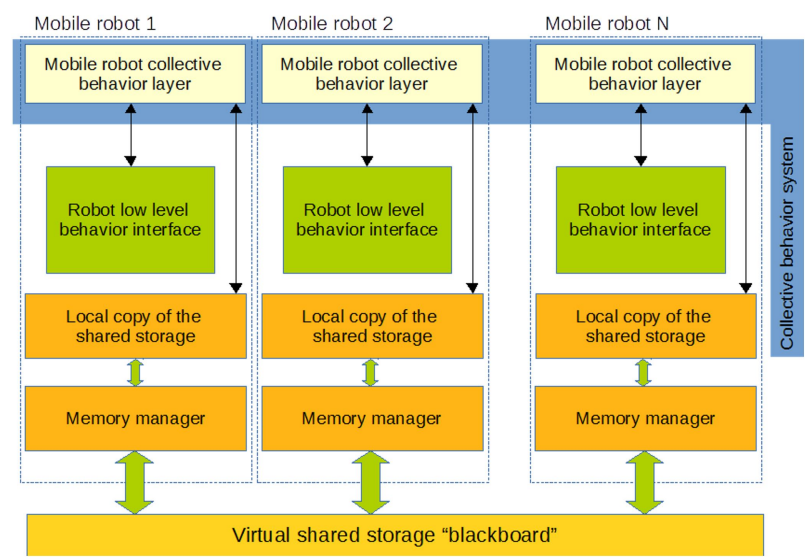

Figure 2: System architecture and shared memory.

We denote it "blackboard" [16]. In order to simplify the synchronization task, it is being done multiple times per every iteration of the tessellation algorithm and thus local "blackboards" are considered to be equalized.

The algorithm starts with the update of robots coordinates $\boldsymbol{R}$, which are filled into the "blackboard" by each robot of the group:

$$
R[j]=\left[\begin{array}{c}
x_{1}[j], y_{1}[j] \\
\vdots \\
x_{N}[j], y_{N}[j]
\end{array}\right]
$$

where $N-$ is the number of robots and $j=0 \ldots T, t=\Delta t \cdot j$ is a transformation into discrete time.

After this step a Voronoi tessellation $V_{i}=$ $\left\{P=(x, y) \in Q, d\left(P, P_{k}\right) \leq d\left(P, P_{l}\right), k \neq l\right\}, \quad$ where $d$ - denotes the distance function and $P_{k, l}$ are subsets in $\boldsymbol{Q}$, is built-up $[17,18]$ locally on each robot computational unit and is then verified by direct comparison via publishing the results onto the "blackboard".

Voronoi tessellation has vertices located in infinity and thus must be cut in order to create a structure within convex territory border $\partial Q$, which is described by a polygon with $M$ vertices:

$$
\partial Q=\left[\begin{array}{c}
B_{x}[0], B_{y}[0] \\
\vdots \\
B_{x}[M], B_{y}[M]
\end{array}\right]
$$

In order to cut infinity vertices away, there is a following method used. Tessellation function returns vectors showing directions to the infinity vertices. A substitute vertex for each infinity vertex is placed on a distance, which is bigger than $\max (\operatorname{sizeof}(\partial Q)$ ) (see Figure 3). The Vatti clipping algorithm $[19,20]$ is executed afterwards in order to limit the final tessellation $V_{i}$ by $\partial Q: V_{i}^{\prime}=V_{i} / \partial Q$.

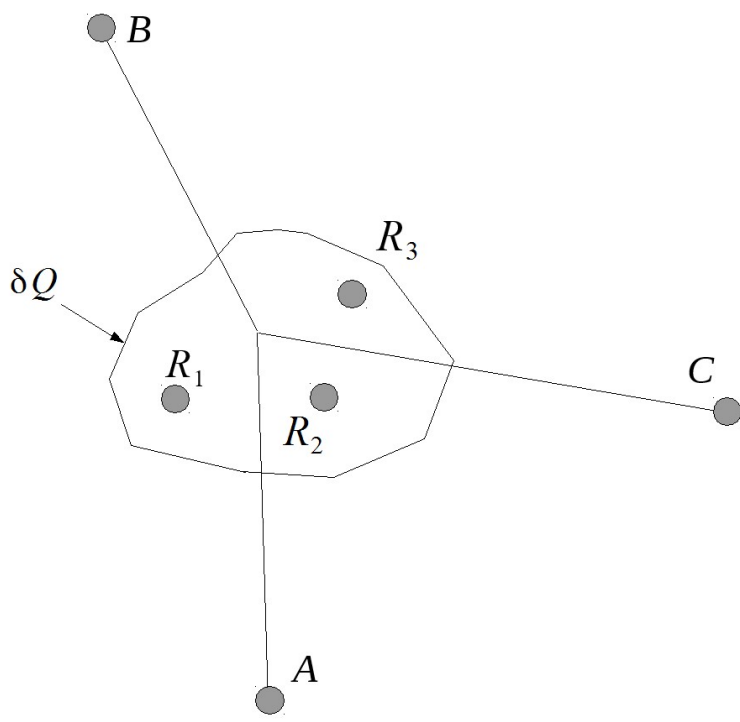

Figure 3: Example of tessellation for three robots.

The verification of the results, which is generated locally on each robot is done after the tessellation is ready. Results of the algorithm execution are shown in Figure 4. Afterwards, the area and corresponding centroid coordinates are calculated as follows:

$$
A_{j}=\frac{1}{2} \sum_{i=0}^{i=G_{N}[j]-1}\left(G x_{[j, i]} \cdot G y_{[j, i+1]}-G x_{[j, i+1]} \cdot G y_{[j, i]}\right),
$$

$$
\begin{aligned}
P[j]_{X}= & \frac{1}{6 A} \sum_{i=0}^{i=G_{N}[j]-1}\left(G x_{[j, i]}+G x_{[j, i+1]}\right) \\
& \cdot\left(G x_{[j, i]} \cdot G y_{[j, i+1]}-G x_{[j, i+1]} \cdot G y_{[j, i]}\right), \\
P[j]_{y}= & \frac{1}{6 A} \sum_{i=0}^{i=G_{N}[j]-1}\left(G y_{[j, i]}+G y_{[j, i+1]}\right) \\
& \cdot\left(G x_{[j, i]} \cdot G y_{[j, i+1]}-G x_{[j, i+1]} \cdot G y_{[j, i]}\right),
\end{aligned}
$$

where $G[j]=\left[G x_{[j, i]}, G y_{[j, i]}\right], i=0 \ldots G_{N}[j]$ - are coordinates of the $V_{j}^{\prime}$ borders for $j$-robot, $G_{N}[j]$ is a quantity of vertices for the $V_{j}^{\prime}, A_{j}$ - the area of $V_{j}^{\prime}$, and $P[j]_{x}, P[j]_{y}-$ coordinates of $V_{j}^{\prime}$ centroid $P_{j}$. Calculated centroid $P_{j}$ becomes a target point for the robot-positioning algorithm and thus after algorithm execution the whole area is divided into subspaces $V_{j}^{\prime}$ and robots are positioned in $P_{j}$. Based on the Voronoi tessellation definition, it is claimed that the "virtual borders" of $V_{j}^{\prime}$ are separating all robots from the very first step of the algorithm. 


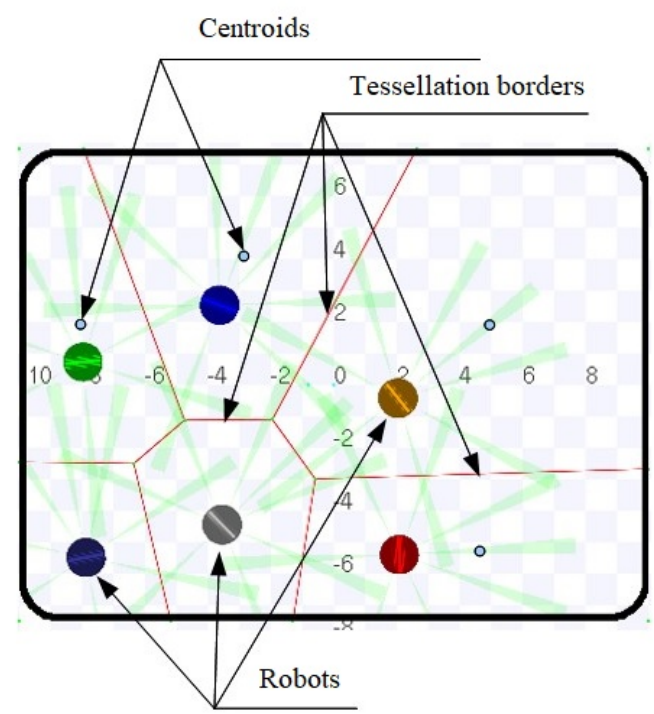

Figure 4: Results of the algorithm execution.
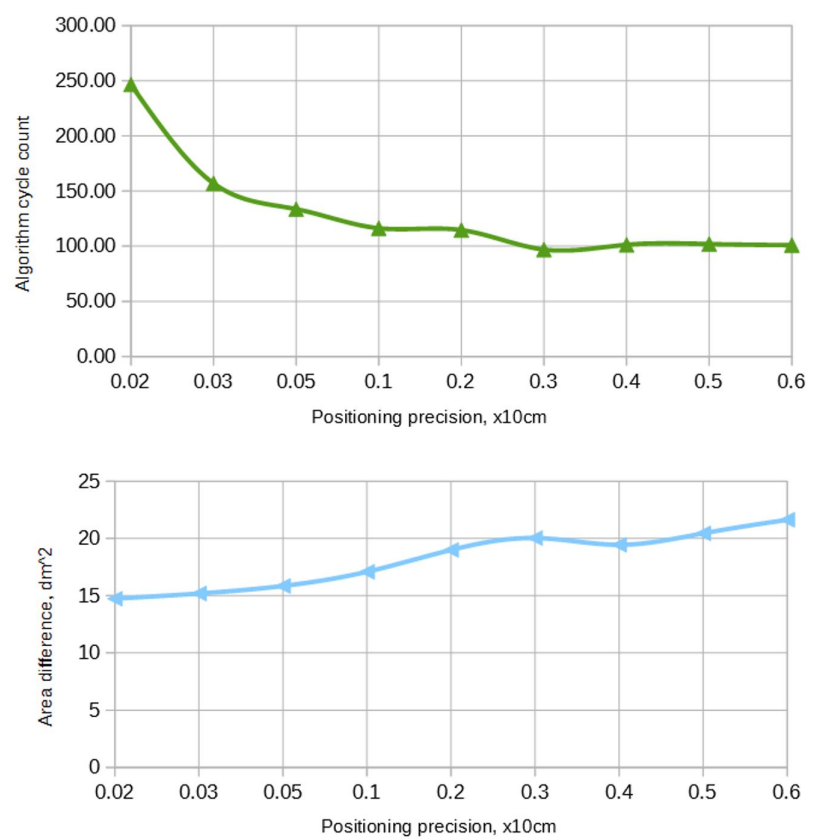

Figure 5: Algorithm properties versus positioning precision.

\section{Parametrization}

The target point $P_{j}$ for the robot is being updated every $T_{i}$, so this point can be thought as running away all the time until robot reaches it. The reach definition is based on the positioning precision parameter $\partial P$ - threshold for the robot position relatively target point $P_{j}$.
Update interval period $T_{i}$ is more or less a technical parameter, which is heavily dependent on hardware capabilities of the robotic system. For further experiments, this parameter is fixed to $T_{i}=100 \mathrm{~ms}$ value. The positioning precision $\partial P$ is a parameter that defines how long it would take the algorithm to converge and as well defines the final "quality" of tessellation, i.e. deviation of the robot position from the ideal centroid point.

Based on simulation data (Figure 5) the positioning precision $\partial P$ is fixed on $1 \mathrm{~cm}$ for all further experiments.

\section{Properties study and simulation}

The simulation is done for the robotic group, except robot number dependency study, containing 6 robots of $12 \mathrm{~cm}$ size with $5 \mathrm{~cm} / \mathrm{s}$ linear velocity and $0.75 \mathrm{rad} / \mathrm{sec}$ angular velocity. The polygon is rectangular shape with size of $2 \times 1.5 \mathrm{~m}$ unless otherwise noted.

One of the quality measure for the partitioning algorithm is an area difference between the smallest and biggest parts $\Delta A$, but as well relative area difference shall be tracked $\partial A=\frac{\Delta A}{\max \left(A_{j}\right)}, j=1 \ldots N$, where $A_{j}$ is an area for the part $j$. Tests showed that values $\Delta A$ and $\partial A$ are not necessarily associated. For instance, when the number of robots fluctuates, the absolute value stays approximately stable, whereas relative difference grows, since the parts are getting smaller when a robot is added (see Figure 6).

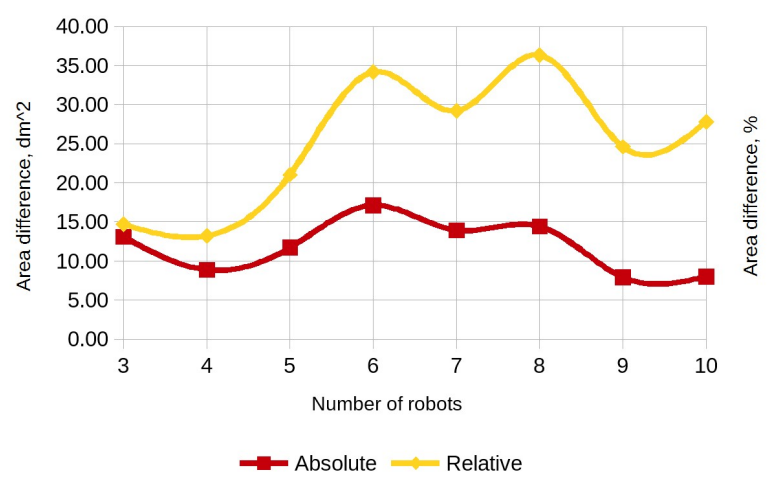

Figure 6: Absolute and relative area difference versus number of robots.

The time of the experiment (as a cycle count) as well as both $\Delta A$ and $\partial A$ were logged during the simulation. As variable parameters, the number of robots, robotic group original position and the test polygon borders were taken. 


\subsection{Experiment I: number of robots}

When the number of robot variation all three different start configurations (see Figure 7) for robotic group were considered, the average values and deviations were calculated. The results of robots quantity variation are shown in Figure 8 while final configurations are shown in Figure 9.

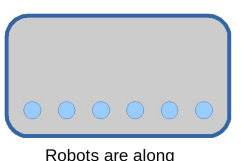

Robots are along
the longer side

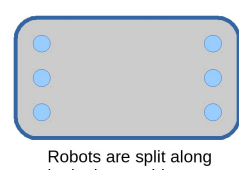

Robots are split alon
both shorter sides

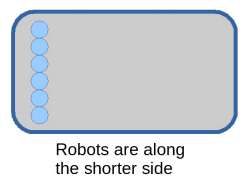

Figure 7: Robotic group start configurations.

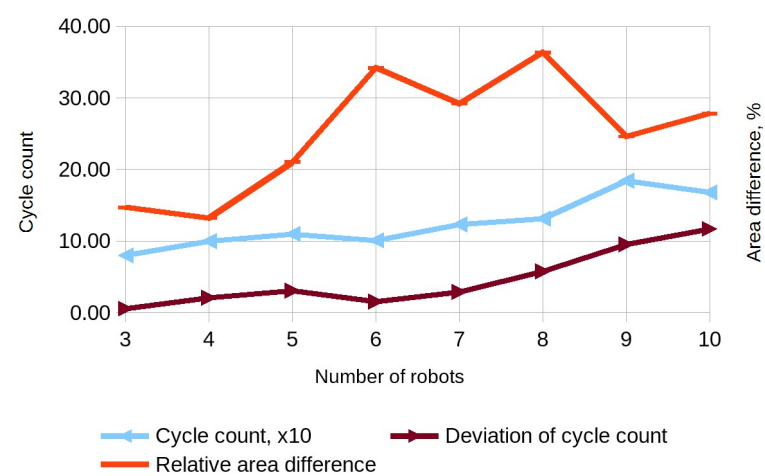

Figure 8: Cycle count average and its deviation in the experiment of numbers of robots variation.

It is clear, that cycle count is growing with the number of robots. Here it is important to notice, that the cycle count is a measure of the duration, but not the algorithm complexity in this case. The deviation vale grows as well, which says that each additional robot not only slows down the process of tessellation, but also brings an uncertainty to the result. As per definition, the Voronoi tessellation is not a unique configuration and thus the is a possibility to get several tessellation configurations based on centroids within given borders [21]. There were traces of robots operation saved during the experiment in order to investigate smoothness of the travel during tessellation. The traces are shown in Figure 10 for the case of 6 robots. As one can see, robots with the smallest initial area had to change direction of movement in order to accomplish the tessellation.
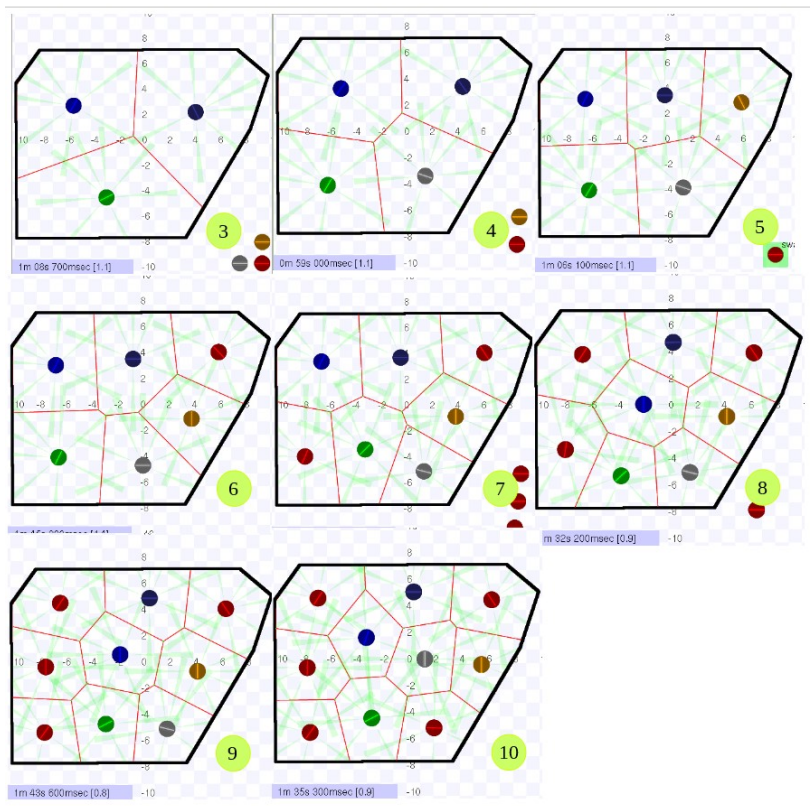

Figure 9: Final configurations for Experiment I.

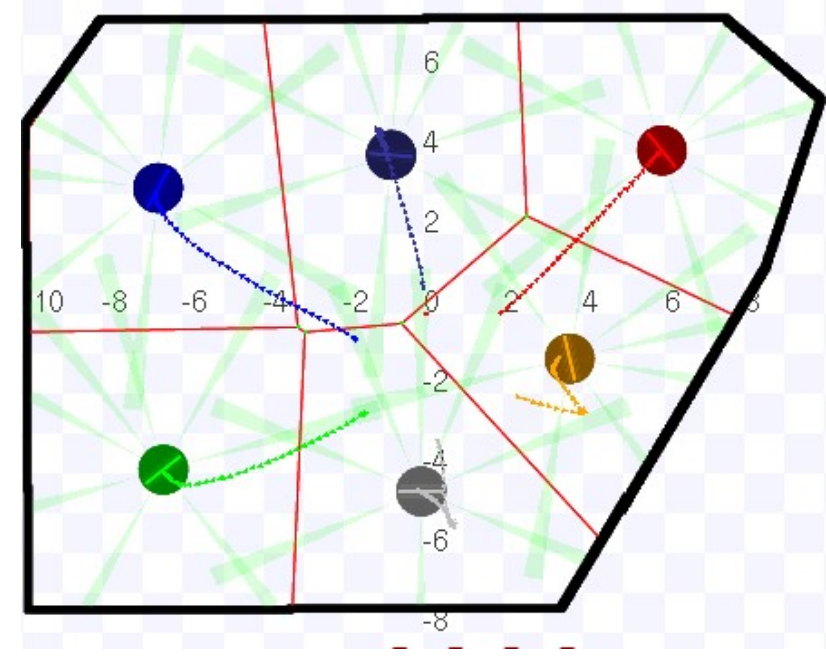

Figure 10: Algorithm run traces.

\subsection{Experiment II: start position variation}

The group start position impact analysis was done by shifting the entire group within II quadrant of the polygon coordinate system (see Figure 11). The gathered data of the cycle count and area difference are shown in Figure 12 as a surface graph. Moving the group towards the polygon center equalize the areas of tessellation, despite the duration grows. 


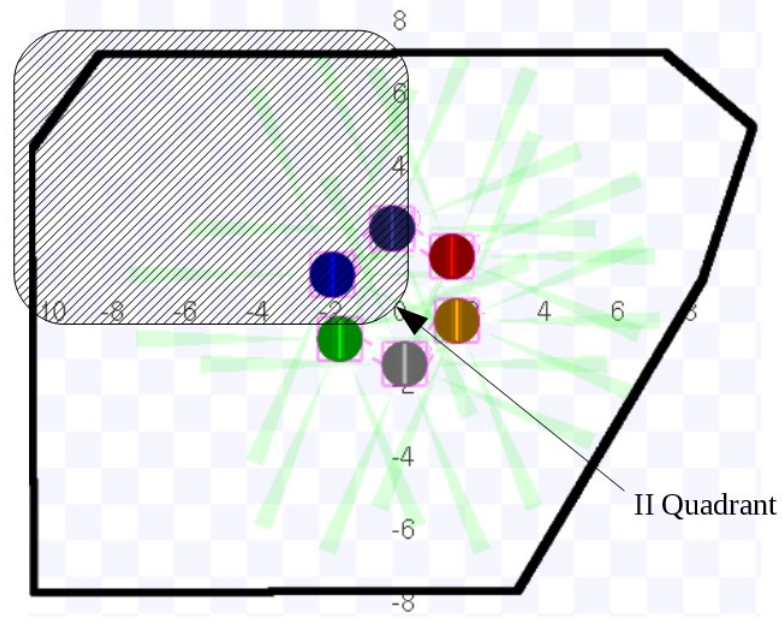

Figure 11: Robotic group start position shift study setup.

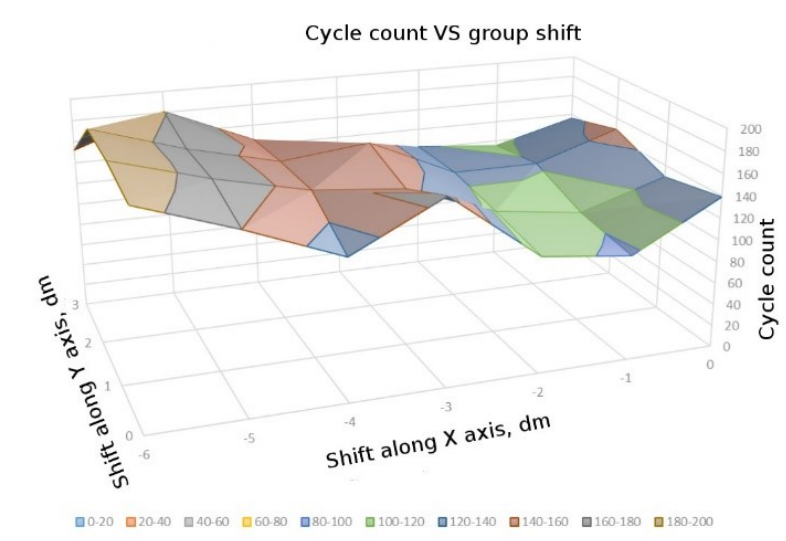

Relative area difference VS group shift

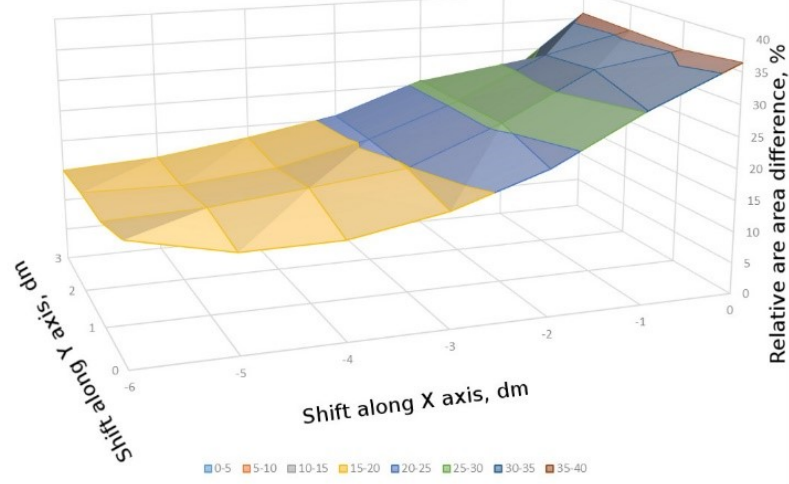

Figure 12: Robotic group start position shift study results.

\subsection{Experiment III: border variation}

Different border shapes were considered for the study of the border variation influence on the tessellation algorithm. This experiment was performed with a fixed number of robots (6) and the fixed start configuration (robots
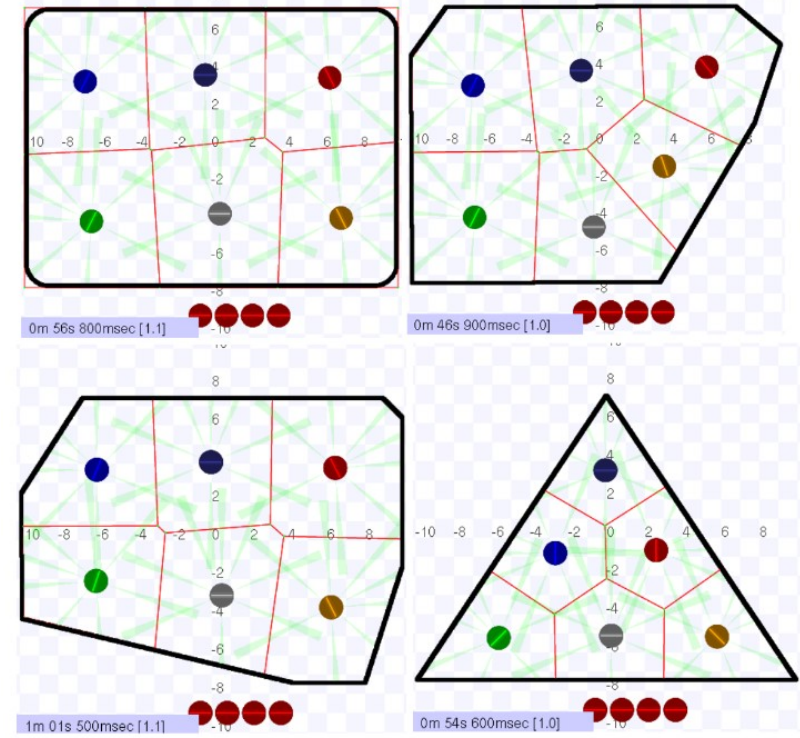

Figure 13: Border shape influence study final configurations.

are grouped around the center of the polygon). The border shapes and the results are shown in Figure 13.

\section{Conclusion and future work}

This paper is focused on the particular Voronoi tessellation-based approach of robotic group activity coordination. Despite the idea of algorithm has been considered previously $[10,11]$, there were still many open points challenging the practical implementation of the method. System architecture development, algorithm of Voronoi infinity-located vertices parsing, algorithm structure and communication flow allowing continuous execution of tessellation routine, clear parametrization including positioning error and investigation of algorithm properties are among of most important points of the article. Based on the data, gathered during the experiments, we can say that:

- Experiments show that the algorithm is robust against variations of the start position, border shape and number of robots;

- Tessellation is done in a way which guarantees a personal subspace for each robot and the difference between subspace areas are $15 . . .35 \%$;

- The cycle count of the algorithm grows with the number of robots in the group. 
It is important to notice that at the end of the algorithm operation, each robot is located in the center of the associated area and thus the following operation may be optimized using the information about known start position.

There are two main drawbacks of the proposed solution: positioning information being required from the global navigation system, polygon border required to be convex. Our future works will naturally focus on resolution of these points. Considering that having position information is quite challenging, authors are looking into direction of joint use of navigation and mapping algorithms (such as SLAM) and the proposed coordination algorithm in future works. Since the algorithm is created as part of area coverage solution, one of the possible ways of development is about coverage "interest" dependent tessellation (with a given or dynamically distributed density function). For example, this problem is important for cleaning task where the dirt amount is defined as a density function on the polygon and either given or estimated using information from previous cleaning runs.

Acknowledgement: This study was partially supported by RFBR grant number 16-01-00429 A. Authors are grateful for the support and assistance.

\section{References}

[1] A. Nikitenko, J. Grundspenkis, A. Liekna, M. Ekmanis, G. Kulikovskis, I. Andersone, Multi-robot system for vacuum cleaning domain, In: Y. Demazeau, F. Zambonelli, J. M. Corchado, J. Bajo (Eds.), Advances in Practical Applications of Heterogeneous Multi-Agent Systems (PAAMS 2014), Lecture Notes in Computer Science, Springer, Cham, 8473, 363-366

[2] M. Lindhé, P. Ögren, K. H. Johansson, Flocking with obstacle avoidance: a new distributed coordination algorithm based on Voronoi partitions, Robotics and Automation, Proceedings of the IEEE ICRA 2005, DOI 10.1109/ROBOT.2005.1570372

[3] D. Portugal, R. Rocha, A survey on multi-robot patrolling algorithms, In: L. M. Camarinha-Matos (Ed.), Technological Innovation for Sustainability, DoCEIS 2011, IFIP Advances in Information and Communication Technology, Springer, Berlin, Heidelberg, 349, 139-146

[4] A. Pennisi, F. Previtali, F. Ficarola, D. D. Bloisi, L. locchi, A. Vitaletti, Distributed sensor network for multi-robot surveillance, Procedia Computer Science, 2014, 32, 1095-1100, DOI 10.1016/j.procs.2014.05.538

[5] V. Alexandrov, K. Kirik, A. Kobrin, A hardware-based modeling approach for real world collaborative multi-robot tasks, Paladyn, Journal of Behavioral Robotics, DOI 10.1515/pjbr-20160003

[6] V. Alexandrov, A. I. Kobrin, The architecture of mobile robot, a part of software and hardware system for group control algorithms development, Radioelectronics Journal (Online), 2017,
ISSN 1684-1719, http://jre.cplire.ru/jre/may11/8/text.pdf

[7] V. Gorodetskiy, O. Karsaev, V. Samoilov, V. Skormin, Multi-agent technology for air traffic control and incident management in airport airspace, Proceedings of the International Workshop "Agents in Traffic and Transportation", Estoril, Portugal, IEEE Computer Press, 2008, 119-125

[8] J. van den Berg, J. Snoeyink, M. Lin, D. Manocha, Centralized path planning for multiple robots: Optimal decoupling into sequential plans, Conference: Robotics: Science and Systems V, University of Washington, Seattle, USA, 2009, DOI 10.15607/RSS.2009.V.018

[9] E. Bonabeau, M. Dorigo, G. Theraulaz, Swarm intelligence: from natural to artificial systems, Santa Fe Institute Studies on the Sciences of Complexity, Oxford Press, 1999

[10] J. Cortés, S. Martínez, T. Karatas, F. Bullo, Coverage control for mobile sensing networks, IEEE Transactions on Robotics and Automation, 2004, 20(2), 243-255, DOI 10.1109/TRA.2004.824698

[11] A. Breitenmoser, M. Schwager, J.-C. Metzger, R. Siegwart, D. Rus, Voronoi coverage of non-convex environments with a group of networked robots, Proceedings of the IEEE International Conference on Robotics and Automation, 2010, 49824989, DOI 10.1109/ROBOT.2010.5509696

[12] S. G. Lee, Y. Diaz-Mercado, M. Egerstedt, Multirobot control using time-varying density functions, IEEE Transactions on Robotics, 2015, 31(2), 489-493, DOI 10.1109/TRO.2015.2397771

[13] P. Bhattacharya, M. L. Gavrilova, Voronoi diagram in optimal path planning, 4th International Symposium on Voronoi Diagrams in Science and Engineering (ISVD 2007), Glamorgan, 2007, 38-47, DOI 10.1109/ISVD.2007.43

[14] W. Sun, L. Dou, J. Chen, H. Fang, A multi-robot target tracking algorithm with centroidal Voronoi tessellation and consensus strategy, Proceedings of the 29th Chinese Control Conference, Beijing, 2010, 4607-4612

[15] Q. Du, V. Faber, M. Gunzburger, Centroidal Voronoi tessellations: applications and algorithms, Journal SIAM Review, 1999, 41(4), 637-676

[16] TABLE_VORONOI: Voronoi Diagram Data [Online], http://people.sc.fsu.edu/ jburkardt/f_src/table_voronoi/ table_voronoi.html

[17] J. Burkardt, M. Gunzburger, J. Peterson, R. Brannon, User manual and supporting information for library of codes for centroidal Voronoi point placement and associated zeroth, first and second moment determination, Technical Report, SAND20020099, Sandia National Laboratories, February, 2002

[18] General Polygon Clipper library [Online], http://www.cs.man. ac.uk/ toby/alan/software/index.html

[19] R. Vatti Bala, A generic solution to polygon clipping, Communications of the ACM, ACM New York, NY, USA, 1992, 35(7), 56-63

[20] J. Burns, Centroidal Voronoi tessellations, Whitman College Academic, Walla Walla, WA, 2009, https://www.whitman.edu/ Documents/Academics/Mathematics/burns.pdf

[21] R. van Liere, J. Harkes, W. de Leeuw, A distributed blackboard architecture for interactive data visualization, Proceedings Visualization '98, Research Triangle Park, NC, USA, 1998, 225-231, DOI 10.1109/VISUAL.1998.745307 